Apidologie, 1978, 9 (2), 117-122.

\title{
INHALT DER HONIGBLASE DER ARBEITSBIENEN VON APIS MELLIFICA L. WÄHREND DER TANNENHONIGTAUTRACHT
}

\section{Contenu du jabot des ouvrières Apis mellifica $\mathbf{L}$. pendant la miellée de miellat de sapin blanc}

\author{
Zofia DEMIANOWICZ \\ Institut für Naturwissenschaftliche Grundlagen der Pflanzenproduktion \\ Landwirtschaftliche Akademie Lublin (Pologne)
}

\author{
SUMMARY \\ THE CONTENTS OF THE HONEY SAC OF THE WORKER BEES \\ OF A PIS MELLIFICA L. DURING THE HONEYDEW FLOW OF THE SILVER FIR
}

\begin{abstract}
During the honeydew flow the returning foraging bees were caught at the flight board. Their honey sacs were prepared out and weighed, and then the dry substance of the contents was evaluated by means of a refractometer. From another part of the out prepared honey sacs the contents were analysed by the microscope. A negative correlation resulted between the amount of honeydew collected by the bees (ascertained by the honey sac weight) and the percentage of the dry substance in the honeydew. Besides it was found that the bees brought with them fungus spores, which develop on the honeydew (Fumago vagans and Helminthosporium sp., very rare Tripospermum pinophilum and Capnophialophora pinophila).
\end{abstract}

Besides honeydew, large amounts of water were foraged by the bees, especially in the midday hours.

The results of the tests are shown in Table 1 and in the figures 1 and 2.

\section{ZUSAMMENFASSUNG}

Während der Honigtautracht wurden die heimkehrenden Trachtbienen am Flugbrett abgefangen. Ihre Honigblasen wurden herauspräpariert und gewogen, und anschliessend wurde der Trockensubstanzgehalt des Inhaltes mit dem Refraktometer bestimmt. Der Inhalt von einem anderen Teil der herauspräparierten Honigblasen wurde mikroskopisch untersucht. Es ergab sich eine negative Korrelation zwischen der Menge des von Bienen eingetragenen Honigtaus (ermittelt als Honigblasengewicht) und dem Prozentgehalt der Trockensubstanz im Honigtau. Ausserdem wurde festgestellt, dass von den Bienen auch Pilzsporen mitgebracht wurden, die sich auf dem Honigtau entwickeln (Fumago vagans und Helminthosporium sp., sehr selten Tripospermum pinophilum und Capnophialophora pinophila). 
Ausser Honigtau wurden von den Bienen, insbesondere in den Mittagsstunden, grosse Wassermengen eingetragen.

Die Untersuchungsergebnisse sind in Tabelle 1 und den Abbildungen 1 u. 2 dargestellt.

\section{EINLEITUNG}

Mit dem Problem des Honigtaus und der Honigtauhonige beschäftige ich mich seit 1966. In Zusammenarbeit mit Mykologen ist es mir gelungen, die Pilzflora näher zu bearbeiten, die sich auf dem Tannenhonigtau entwickelt, als auch viele Pilzarten zu bestimmen, die in Honigtauhonigen anzutreffen sind. $\mathrm{Zu}$ Beginn meiner Arbeit war es für mich auch von grossen Interesse, was die Bienen mit dem Honigtau in den Stock mitbringen. Die Ergebnisse dieser Untersuchungen möchte ich in der vorliegenden Arbeit besprechen.

\section{METHODE}

Die Untersuchungen wurden im Swietokrzyski-Nationalpark durchgeführt, dessen bewaldete Fläche 5810 ha beträgt. Der Park umfasst ein Gebiet alter, wenig hoher Berge, die vorwiegend mit Tannenwäldern bewachsen sind.

Die Untersuchungen wurden im Zeitraum von 2 Jahren - 1966 und 1967 - auf einem Bienenstand durchgeführt, der tief im Walde lag.

Bei günstigen Wetterverhältnissen wurden die von den Sammelflügen heimkehrenden Bienen im ersten Jahr je eine Stunde und im zweiten Jahr je zwei Stunden lang am Flugbrett abgefangen. Die Zahl der jeweils abgefangenen Bienen, deren Honigblasen präpariert wurden, betrug wenigstens 10 Individuen. Im Jahre 1966 wurden diese Untersuchungen während 4 Augusttagen, im Jahre 1967 während 4 Juliund 3 Augusttagen durchgeführt. Die herauspräparierten Honigblasen wurden gewogen und anschliessend wurde der Trockensubstanzgehalt im Honigtau mittels des Refraktometers von Abbe bestimmt. Der Inhalt von einem Teil der Honigblasen wurde auch mikroskopisch untersucht.

\section{ERGEBNISSE}

\section{A. - Honigblasengewicht und Trockensubstanz}

Im Juli 1967 schwankte das Gewicht der Honigblasen zwischen 18,0 und 77,0 mg und betrug durchschnittlich 43,6 mg (Tab. 1). Der Trockensubstanzgehalt im Honigtau schwankte in den Grenzen von 21,0 bis $78,3 \%$ und betrug durchschnittlich $63,1 \%$. Damit hat sich dieser Gehalt als sehr hoch erwiesen. 
TAB. 1. - Honigtaugehalt der Honigblase und Honigtautrockensubstanz.

TABL. 1 - Teneur du jabot en miellat et substance sèche du miellat.

\begin{tabular}{|c|c|c|c|c|c|c|c|c|c|c|c|c|}
\hline \multirow{4}{*}{$\begin{array}{c}\text { Jahr } \\
\text { Année }\end{array}$} & \multicolumn{6}{|c|}{$\begin{array}{l}\text { Honigblasengewicht } \\
\text { in mg } \\
\text { Poids du jabot } \\
\text { en mg }\end{array}$} & \multicolumn{6}{|c|}{$\begin{array}{l}\text { Honigtautrockensubstanz } \\
\text { in \% } \\
\text { Substance sèche du miellat } \\
\text { en } \%\end{array}$} \\
\hline & \multicolumn{6}{|c|}{$\begin{array}{l}\text { Monate } \\
\text { Mois }\end{array}$} & \multicolumn{6}{|c|}{$\begin{array}{c}\text { Monate } \\
\text { Mois }\end{array}$} \\
\hline & \multicolumn{3}{|c|}{ VII } & \multicolumn{3}{|c|}{ VIII } & \multicolumn{3}{|c|}{ VII } & \multicolumn{3}{|c|}{ VIII } \\
\hline & Min. & Max. & $\overline{\mathrm{X}}$ & Min. & Max. & $\bar{X}$ & Min. & Max. & $\dot{\mathrm{X}}$ & Min. & Max. & $\overline{\mathrm{X}}$ \\
\hline $\begin{array}{l}1966 \ldots \\
1967 \ldots\end{array}$ & $-\overline{18,0}$ & $\overline{77,0}$ & $\overline{43,6}$ & $\begin{array}{l}11,0 \\
12,5\end{array}$ & $\begin{array}{l}53,0 \\
60,0\end{array}$ & $\begin{array}{l}24,3 \\
25,5\end{array}$ & $-\overline{21,0}$ & $\overline{78,3}$ & $-\overline{33,1}$ & $\begin{array}{c}5,25 \\
14,0\end{array}$ & $\begin{array}{l}64,4 \\
75,0\end{array}$ & $\begin{array}{l}42,2 \\
42,5\end{array}$ \\
\hline
\end{tabular}

Das Gewicht der Honigblasen schwankte im August 1966 zwischen 11,0 und $53 \mathrm{mg}$. Der berechnete Durchschnittswert betrug $24,3 \mathrm{mg}$. Der Gehalt an Honigtautrockensubstanz schwankte von 5,25\% (um $6 \mathrm{Uhr}$ morgens nach einem Gewitter am Tag zuvor) bis $64,4 \%$ und betrug durchschnittlich $42,2 \%$. Im August 1967 lag das Gewicht der Honigblasen zwischen $12,5 \mathrm{mg}$ und $60,0 \mathrm{mg}$ und betrug durchschnittlich $25,5 \mathrm{mg}$. Der Trockensubstanzgehalt im Honigtau schwankte zwischen 14,0 und $75,0 \%$. Der berechnete Durchschnittswert betrug ähnlich wie im Jahre $196642,5 \%$.

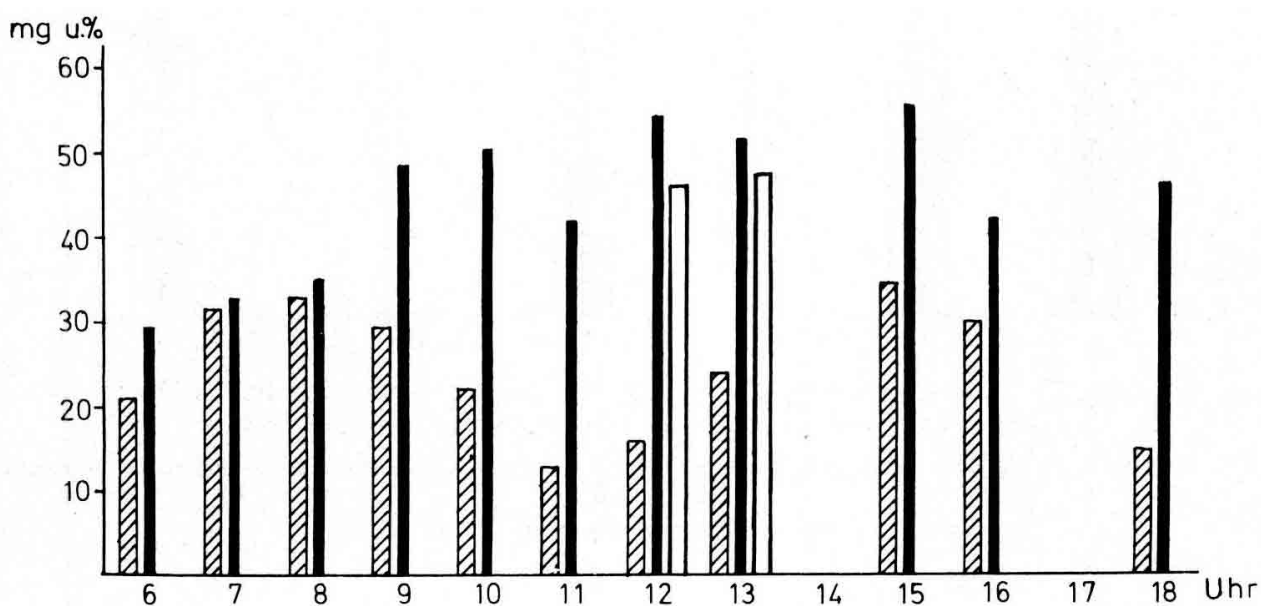

Aвв. 1. - Gewicht der Honigblase, gefüllt mit Honigtau $\square$ oder mit Wasser $\square$ und Prozentgehalt der Honigtau-Trockensubstanz 1 . Jahr 1966.

FIG. 1. - Poids en mg du jabot rempli de miellat $\oslash$ ou d'eau $\square$ et substance sèche du miellat en pourcentage

selon les différentes heures de la journée. Année 1966. 
Schon an der Färbung der Honigblase war der Inhalt zu erkennen : Honigblasen, die Wasser enthielten, waren farblos. Enthielten sie Honigtau, so waren sie dunkel. Honigblasen mit dem Nektar des Weissklees hatten eine leicht gelbliche und die mit dem Nektar vom Heidekraut eine dunkelgelbe Farbe.

Aus den Abb. 1 und 2 ist deutlich zu ersehen, dass eine umgekehrte Abhängigkeit zwischen der von den Bienen geholten Honigtaumenge (Honigblasengewicht mit Honigtau) und dem Prozentgehalt der Honigtautrockensubstanz besteht. Im Laufe des Tages, insbesondere zwischen 10 und $13 \mathrm{Uhr}$, trocknet der Honigtau ein. Dann stieg die Zahl der Wasserholer an. 1966 wurde erst in den wärmeren Mittagsstunden und 1967 den ganzen Tag lang von Bienen Wasser geholt (Abb. 1). Es gab Zeiträume, zu denen die Bienen fast ausschliesslich Wasser eintrugen (Abb. 2). Das Gewicht der Wasser enthaltenden Honigblasen war im Jahre 1967 hoch und betrug 45-55 mg, gelegentlich sogar über $60 \mathrm{mg}$.

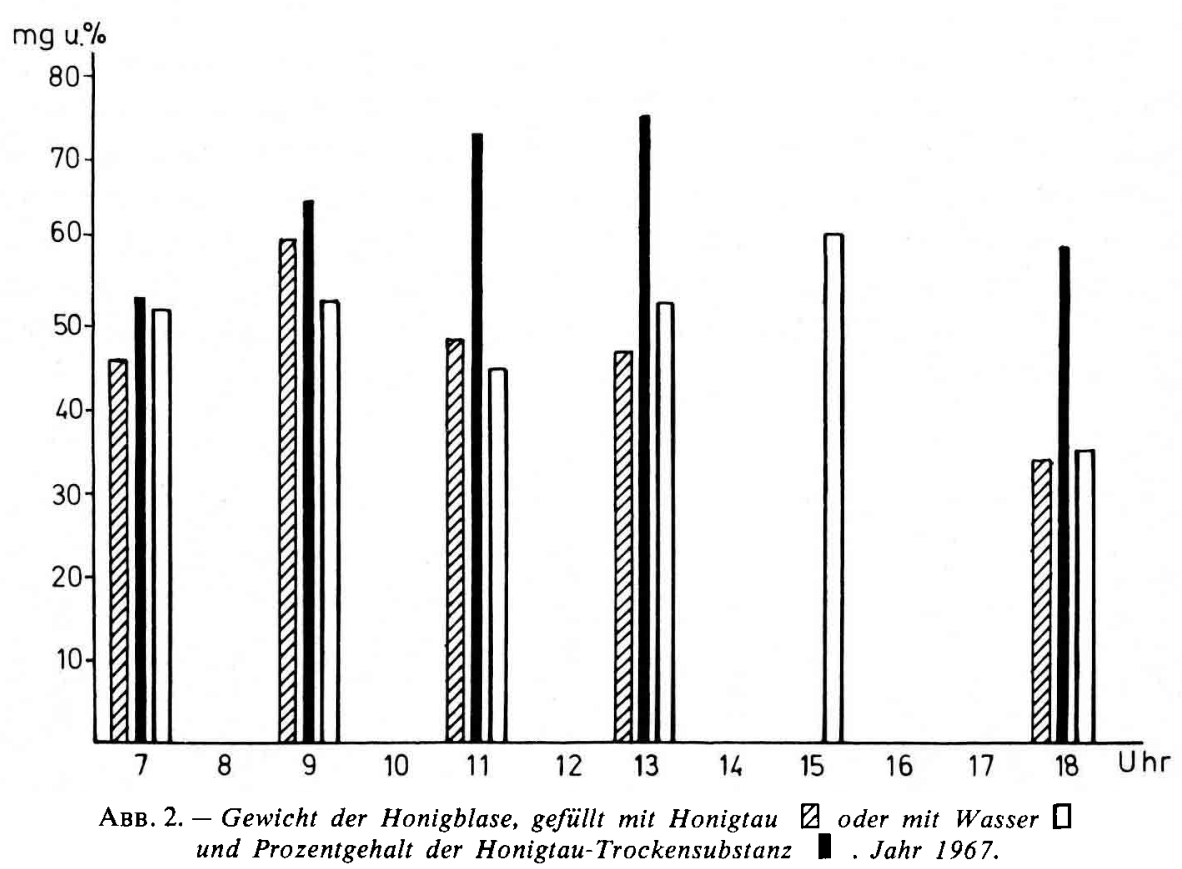

FIG. 2. - Poids en mg du jabot rempli de miellat $\square$ ou d'eau $\square$ et substance sèche du miellat en pourcentage

selon les différentes heures de la journée. Année 1967.

In die Diagramme wurde auch das Gewicht des Wassers eingetragen, das von Bienen schon ab 7 Uhr morgens von einer unweit gelegenen Quelle geholt wurde, die breit auf einer Wiese floss. Im Wasser aus den Honigblasen wurde die Anwesenheit lebender Individuen von Euglene sp. und der Blaualge Nostoc festgestellt. 


$$
\text { B. - Pilze }
$$

Von den Pilzarten, die auf dem Tannenhonigtau vorkamen, wurden im Honigblaseninhalt Sporen von Cladosporium, Fumago vagans und Helminthosporium sp. und gelegentlich auch Sporen und Myzel von Torula sp. angetroffen. Seltener wurden im Honigblaseninhalt Sporen von Tripospermum pinophilum und Capnophialophora pinophila gefunden, die nicht so oft Sporen bilden, obwohl sie ein grosses Myzel bilden.

Eingegangen im Oktober 1977. Reçu pour publication on octobre 1977.

\section{RÉSUMÉ}

Au cours de la miellée de miellat les abeilles butineuses rentrant à la ruche ont été capturées sur la planche de vol. On a extrait et pesé leur jabot et estimé à l'aide d'un réfractomètre la teneur en substance sèche du contenu du jabot. Une autre partie du contenu a été étudiée au microscope. Il est apparu une corrélation négative entre la quantité de miellat rapportée par les abeilles (obtenue d'aprés le poids du jabot) et le pourcentage de matière sèche dans le miellat. On a établi en outre que les abeilles rapportaient également des spores de champignons qui se développent sur le miellat (Fumago vagans et Helminthosporium sp., très rarement Tripospermum pinophilum et Capnophialophora pinophila). journée.

En plus du miellat les abeilles rapportaient aussi de grandes quantités d'eau, en particulier à la mi-

Les résultats sont représentés dans le tableau 1 et les figures 1 et 2 .

\section{LITERATUR VERZEICHNIS}

Borowska A., Demianowicz Z., 1972 - [Fungi on fir honey-dew.] In polish A cta mycol., 8 (2), 175-189.

Demianowicz Z., Borowska A., Dubik G., Pielka J., 1972. - [Fungi in the fir honey.| In polish Ann. Univ. Mariae-Curie Sklodowska, sectio E, 25 (15), 203-212.

Hammer O., 1953. - Observations sur la sécrétion nectarifère chez le romarin, Rosmarinus officinalis. Rev.fr. Apic., 3, 397. 